\title{
Combined metabolic, phenomic and genomic data to prioritize atrial fibrillation-related metabolites
}

\author{
ZHI-TAO YAN $^{1}$, JIN-MEI HUANG ${ }^{2}$, WEN-LI LUO ${ }^{3}$, JI-WEN LIU $^{4}$ and KANG ZHOU ${ }^{1}$ \\ Departments of ${ }^{1}$ Cardiology, ${ }^{2}$ General Surgery and ${ }^{3}$ Gerontology, The First Affiliated Hospital of the Medical College, \\ Shihezi University, Shihezi, Xinjiang 832000; ${ }^{4}$ Department of Internal Medicine, Affiliated Midong Hospital \\ of the People's Hospital of Xinjiang Uygur Autonomous Region, Urumqi, Xinjiang 830000, P.R. China
}

Received June 26, 2018; Accepted February 14, 2019

DOI: $10.3892 / \mathrm{etm} .2019 .7443$

\begin{abstract}
Metabolites in atrial fibrillation (AF) were characterized to further explore the molecular mechanisms of AF by integrating metabolic, phenomic and genomic data. Gene expression data on AF (E-GEOD-79768) were downloaded from the EMBL-EBI database, followed by identification of differentially expressed genes (DEGs) which were used to construct gene-gene network. Then, multi-omics composite networks were constructed. Subsequently, random walk with restart was expanded to a multi-omics composite network to identify and prioritize the metabolites according to the AF-related seed genes deposited in the OMIM database, the whole metabolome as candidates and the phenotype of AF. Using the interaction score among metabolites, we extracted the top 50 metabolites, and identified the top 100 co-expressed genes interacted with the top 50 metabolites. Based on the FDR $<0.05,622$ DEGs were extracted. In order to demonstrate the intrinsic mode of this method, we sorted the metabolites of the composite network in descending order based on the interaction scores. The top 5 metabolites were respectively weighed potassium, sodium ion, chitin, benzo[a]pyrene-7,8-dihydrodiol9,10-oxide, and celebrex (TN). Potassium and sodium ion possessed higher degrees in the subnetwork of the entire composite network and the co-expressed network. Metabolites such as potassium and sodium ion may provide valuable clues for early diagnostic and therapeutic targets for AF.
\end{abstract}

\section{Introduction}

Atrial fibrillation (AF), a highly prevalent heart disorder with a significant genetic component (1), is regarded as the most common sustained arrhythmia in clinical practice

Correspondence to: Dr Zhi-Tao Yan, Department of Cardiology, The First Affiliated Hospital of the Medical College, Shihezi University, 107 Beier Road, Shihezi, Xinjiang 832000, P.R. China E-mail: yanzhigreen@yeah.net

Key words: sinus rhythm, atrial fibrillation, metabolite, multiomics composite network worldwide, which can result in heart failure and represents an important risk factor for ischemic stroke, thereby leading to significant morbidity and mortality (2-4). A higher rate of adverse outcomes for the elderly are connected with AF. According to literature, arrhythmia has been demonstrated to arise because of the interaction between genetic and acquired risk factors (5). However, the molecular pathogenesis of $\mathrm{AF}$ was not well elucidated, leading to the need of exploring the precise mechanisms of this disease as well as developing novel treatments. Extraction of novel biomarkers for AF is critically important to the understanding and future prevention of this disease.

Metabolites have been regarded as the eventual response of biological systems to the changes of inheritance or environment, whose level directly reflect the physiological status of the body (6). Consequently, selecting disease-associated metabolites is very important for enhancing clinical diagnosis $(7,8)$. Of note, functionally associated metabolites and genes are tended to infer to phenotypically similar disorders. Notably, 'omics' data, for example, metabolic, phenomic and genomic, will offer valuable information for identification of disease risk candidate metabolites. From a biological perspective, a biological system is expressed as a multi-omics network. It is a natural method to combine gene, metabolite and phenotype data to establish a composite network for detecting diseaserelated metabolites, and this combination approach can offer accurate information $(9,10)$. Nevertheless, few attempts have been made to explore the possible involvement of cardiac metabolism in AF and the underlying pathways remain poorly understood.

In the present study, with the aim of further clarifying metabolic processes in the progression of AF and to confirm whether metabolic derangements may exert important functions in contributing to the arrhythmia occurrence, human atrial tissues obtained from the matched cohorts in sinus rhythm (SR) were analyzed to identify and prioritize candidate metabolites by constructing a composite network that combined metabolic, phenomic, and genomic data. Specifically, microarray data selection (E-GEOD-79768) and differentially expressed genes (DEGs) identification were first conducted. Then, a multi-omics composite network by combining six data sets was established. Next, we mapped the AF-related seed genes and known metabolites to the composite network, following 
by the prioritization of candidate metabolite related to AF according to the global functional relations of the composite network using random walk with restart (RWR). Via using the interaction score among metabolites, we extracted the top 50 metabolites and identified the top 100 co-expressed genes interacted with the top 50 metabolites.

\section{Materials and methods}

Acquisition of data set and pretreatment of the original data. The genechip data on AF (E-GEOD-79768) (11) were downloaded from the EMBL-EBI database according to the platform of A-AFFY-44 - Affymetrix GeneChip Human Genome U133 Plus 2.0 [HG-U133_Plus_2]. E-GEOD-79768 included 26 samples including data from patients with AF $(n=14)$, and SR samples as control $(n=12)$.

Subsequently, the original data were pre-processed based on a series of steps including background correction, quantile normalization, perfectmatch/mismatch correction, and mediapolish summarization, and transformation of probe IDs into human gene symbols.

Identification of DEGs. As reported, the change of gene expression reflects the propensity of a given disease. In the present study, detecting DEGs between two groups was performed using Student's t-test. Then, we utilized the multiple test to correct the original P-values based on BenjaminiHochberg (12) using false discovery rate (FDR). DEGs were screened out when the FDR was $<0.05$. These DEGs were used to establish a gene network.

Constructing multi-omics composite network. A composite network was constructed by combing six data, which could be denoted by six networks, namely gene, phenotype, metabolite, phenotype-gene association, gene-metabolite association network, as well as phenotype-metabolite association network.

Gene network construction. In the present study, all proteinprotein interactions (PPIs) of human having combine scores (1,048,576 interactions) were downloaded from the STRING to build the original PPI network. Following removal of the duplicated PPIs, and transformation of proteins into gene symbols, 1,515,370 highly correlated gene-gene interactions among 16,785 genes were identified to construct the background PPI network (combine-score of edges <0.8). Subsequently, the intersection between the 16,785 genes in the background PPI network and DEGs was extracted to construct the informative gene-gene network.

Metabolite network construction. First, a total of 4,994 human metabolites were collected from KEGG, HMDB, Reactome, MSEA (13) and SMPDB (14). Subsequently, metabolitemetabolite associations of human and their corresponding confidence scores were extracted from STITCH (15), where the the 4,994 human metabolites were covered. Finally, a total of 3,764 human metabolites and 74,667 human metabolitemetabolite interactions were obtained.

Phenotype network construction. In the phenotype-phenotype similarity associations (16), there are 5,080 phenotypes and similarity scores across them. The majority of recorded human phenotypes were included in these phenotypes. Based on the phenotype-phenotype similarity associations, we constructed a phenotype network.

Gene-metabolite association network construction. With the goal of extracting human gene-metabolite interactions, we downloaded the chemical and gene associations of human and the corresponding confidence scores from STITCH. After discarding the metabolites that were not included in the metabolite network mentioned above and removing the genes that were not involved in the above gene network, overall 192,763 gene-metabolite interactions were obtained among 12,342 genes as well as 3,278 metabolites.

Phenotype-gene association network construction. We got the phenotype-gene interactions according to the OMIM database. Following removing the phenotypes not appearing in the phenotype network and the genes not covered in the gene network, 2,603 gene-phenotype associations were found between 1,715 genes and 1,886 phenotypes. The weighted score was determined as 1 for every phenotype-gene interaction.

Phenotype-metabolite association network construction. We obtained the phenotype-metabolite associations from the HMDB. Analogously, a total of 664 associations between 388 metabolites and 149 phenotypes were reserved after filtration. Moreover, the weighted score was defined as 1 for every phenotype-metabolite interaction.

Establishment of a multi-omics composite network. To identify and prioritize the potential metabolite, the six networks mentioned above were merged into one weighted composite network. Specific steps were as described in Yao et al (17).

Prioritization of candidate metabolite relying on the multiomics composite network. The corresponding seed genes of AF were ABCC9, GJA5, KCNA5, KCNE2, KCNJ2, KCNQ1, $N P P A, N U P 155, S C N 1 B, S C N 2 B$, and $S C N 5 A$, which were deposited in the database of OMIM. Next, we mapped these seed genes and known disease metabolites to the multi-omics composite network.

In an attempt to obtain the candidate metabolite prioritization from the weighted composite network, the RWR method was expanded to the multi-omics composite network (18). Then, we scored the candidate metabolites and ranked these candidate metabolites based on distance proximity. Based on the interaction score, we identified the top 50 metabolites which were defined as the AF-prioritized metabolites.

Then, based on the top 50 metabolites, co-expressed genes interacted with the top 50 metabolites were identified, and then, we analyzed these co-expressed genes. According to the score distribution, the top 100 co-expressed genes were extracted.

The subnetwork on the top 50 metabolites obtained from the composite network, and the co-expressed network were constructed. Furthermore, degree analyses were performed for these two networks to further identify several important AF-related metabolites. 
Table I. The top 20 differentially expressed genes (DEGs).

\begin{tabular}{lccc}
\hline Gene symbols & FDR & Gene symbols & FDR \\
\hline LBH & $9.23 \mathrm{E}-06$ & IDH3A & $1.73 \mathrm{E}-04$ \\
PPP4C & $7.52 \mathrm{E}-05$ & RNF 141 & $1.85 \mathrm{E}-04$ \\
NOL6 & $7.48 \mathrm{E}-05$ & TOMM22 & $2.14 \mathrm{E}-04$ \\
AGK & $1.25 \mathrm{E}-04$ & TSPAN12 & $2.78 \mathrm{E}-04$ \\
DEDD & $1.37 \mathrm{E}-04$ & PDK1 & $2.92 \mathrm{E}-04$ \\
THOC6 & $1.55 \mathrm{E}-04$ & SAFB & $3.20 \mathrm{E}-04$ \\
COL21A1 & $1.61 \mathrm{E}-04$ & LRIF1 & $3.21 \mathrm{E}-04$ \\
CAPZA2 & $1.65 \mathrm{E}-04$ & INPP4A & $3.26 \mathrm{E}-04$ \\
FLI1 & $1.69 \mathrm{E}-04$ & ZBTB43 & $3.32 \mathrm{E}-04$ \\
KLHL12 & $1.71 \mathrm{E}-04$ & SETD5 & $3.39 \mathrm{E}-04$ \\
\hline
\end{tabular}

FDR, false discovery rate.

Table II. The composite network.

\begin{tabular}{lcc}
\hline $\begin{array}{l}\text { Statistics of the } \\
\text { composite network }\end{array}$ & Nodes & Edges \\
\hline $\begin{array}{l}\text { Gene-gene network } \\
\text { Metabolite-metabolite } \\
\text { network }\end{array}$ & 571 & 4,254 \\
$\begin{array}{l}\text { Phenotype-phenotype } \\
\text { network }\end{array}$ & 3764 & 74,667 \\
$\begin{array}{l}\text { Gene-metabolite } \\
\text { association network }\end{array}$ & 5080 & $10,140,046$ \\
$\begin{array}{l}\text { Phenotype-gene } \\
\text { association network }\end{array}$ & 571 & 3,763 \\
$\begin{array}{l}\text { Phenotype-metabolite } \\
\text { association network }\end{array}$ & 5080 & 2,600 \\
\begin{tabular}{l} 
Sum \\
\hline
\end{tabular} & 537 & 664 \\
\hline
\end{tabular}

\section{Results}

DEG identification and construction of multi-omics composite network. In the present study, we used DEGs to comprise the gene nodes of the composite network, thus, we first identified the DEGs between the two groups. Based on the FDR $<0.05$, a total of 622 genes were extracted as DEGs. The top 20 DEGs are shown in Table I. This method identified and prioritized the disease-related metabolites via combining multi-omics information. In the present study, we first established a multiomics composite network by merging information derived from the genome, phenome, and metabolome. There were three kinds of nodes (metabolite, gene, and phenotype) and six kinds of interactions (gene-gene, phenotype-phenotype, metabolite-metabolite, phenotype-gene, gene-metabolite, and phenotype-metabolite) in the multi-omics composite network. In this network, there were 9,415 nodes and 10,227,292 edges (Table II).
Table III. The top 50 metabolites

\begin{tabular}{|c|c|c|}
\hline Metabolite ID & Metabolite name & Score \\
\hline 813 & Potassium & 0.004268 \\
\hline 923 & Sodium ion & 0.002251 \\
\hline 24139 & Chitin & 0.001765 \\
\hline 41322 & $\begin{array}{l}\text { Benzo[a]pyrene-7,8- } \\
\text { dihydrodiol-9,10-oxide }\end{array}$ & 0.001509 \\
\hline 2662 & Celebrex (TN) & 0.001131 \\
\hline 5957 & Triphosadenine (DCF) & 0.001094 \\
\hline 3676 & Dentipatch (TN) & 0.001053 \\
\hline 935 & Nickel & 0.001025 \\
\hline 187 & Acetylcholine & 0.000897 \\
\hline 1775 & Dilantin (TN) & 0.000829 \\
\hline 2554 & Tegretol (TN) & 0.000805 \\
\hline 5816 & Adrenalin (TN) & 0.000798 \\
\hline 1103 & Spermine & 0.000772 \\
\hline 105024 & L-selenomethionine & 0.000758 \\
\hline 945 & Nitric oxide & 0.000753 \\
\hline 3348 & Telfast (TN) & 0.000735 \\
\hline 888 & Magnesium ion & 0.000725 \\
\hline 24755493 & PIPP & 0.000711 \\
\hline 4913 & Procainamide (INN) & 0.000709 \\
\hline 3446 & Neurontin (TN) & 0.00064 \\
\hline 5505 & Orinase (TN) & 0.00063 \\
\hline 5284627 & Topamax (TN) & 0.000603 \\
\hline 2337 & Parathesin $(\mathrm{TN})$ & 0.000546 \\
\hline 4946 & Propranolol (TN) & 0.000536 \\
\hline 5202 & Serotonin & 0.000535 \\
\hline 439155 & S-adenosylhomocysteine & 0.000521 \\
\hline 2812 & Lotrimin $(\mathrm{TN})$ & 0.000515 \\
\hline 89594 & Habitrol (TN) & 0.000484 \\
\hline 4914 & Procaine (INN) & 0.000483 \\
\hline 24798682 & $\begin{array}{l}\text { 1-Radyl-2-acyl-sn- } \\
\text { glycero-3-phosphocholine }\end{array}$ & 0.00048 \\
\hline 5839 & Aldosterone & 0.00047 \\
\hline 5354618 & Caesium & 0.000439 \\
\hline 5280961 & Genistein & 0.000429 \\
\hline 10114 & Hidermart (TN) & 0.000426 \\
\hline 441074 & Conquinine & 0.000409 \\
\hline 6022 & Adenosine 5'-diphosphate & 0.000395 \\
\hline 444795 & Tretinoin $(\mathrm{TN})$ & 0.00038 \\
\hline 1548943 & Capsaicin (JAN/USP) & 0.000367 \\
\hline 3121 & Depakene $(\mathrm{TN})$ & 0.000364 \\
\hline 702 & Dehydrated ethanol & 0.000361 \\
\hline 5819 & Forthyron (TN) & 0.000342 \\
\hline 172198 & Delivert (TN) & 0.000327 \\
\hline 60961 & Adenocard (TN) & 0.000327 \\
\hline 94715 & Glucuronic acid & 0.000317 \\
\hline 5994 & Prometrium (TN) & 0.0003 \\
\hline 2519 & Caffeine (USP) & 0.000293 \\
\hline 65064 & Epigallocatechin 3-gallate & 0.000273 \\
\hline 8980 & Benadryl (TN) & 0.000271 \\
\hline 5546 & Dyrenium (TN) & 0.000267 \\
\hline 2719 & Chloroquine (USP/INN) & 0.000264 \\
\hline
\end{tabular}




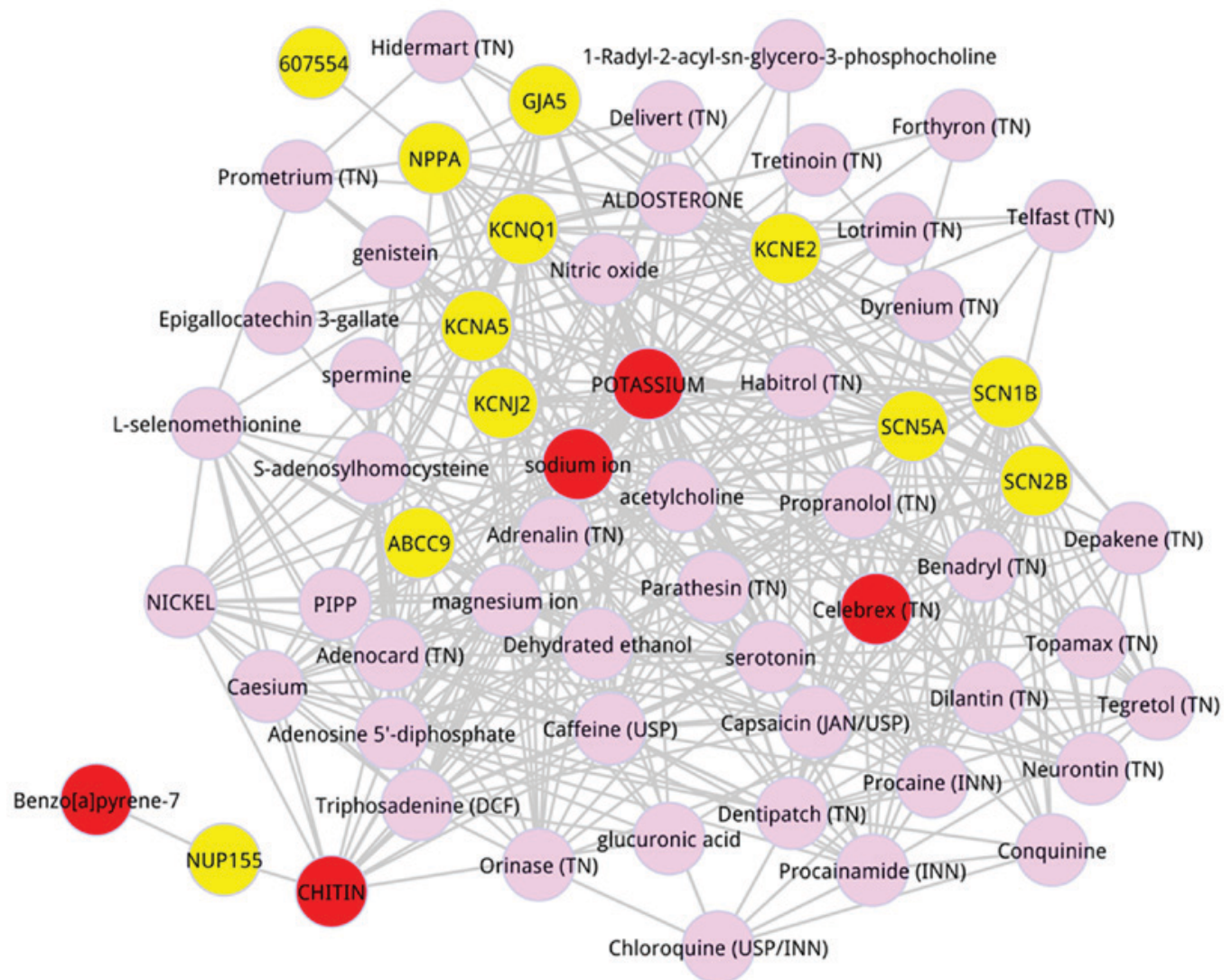

Figure 1. Composite network including the top 50 metabolites and the 11 seed genes. Yellow nodes, the seed genes; Pink nodes, the metabolites; and red nodes, behalf of the top 5 metabolites.

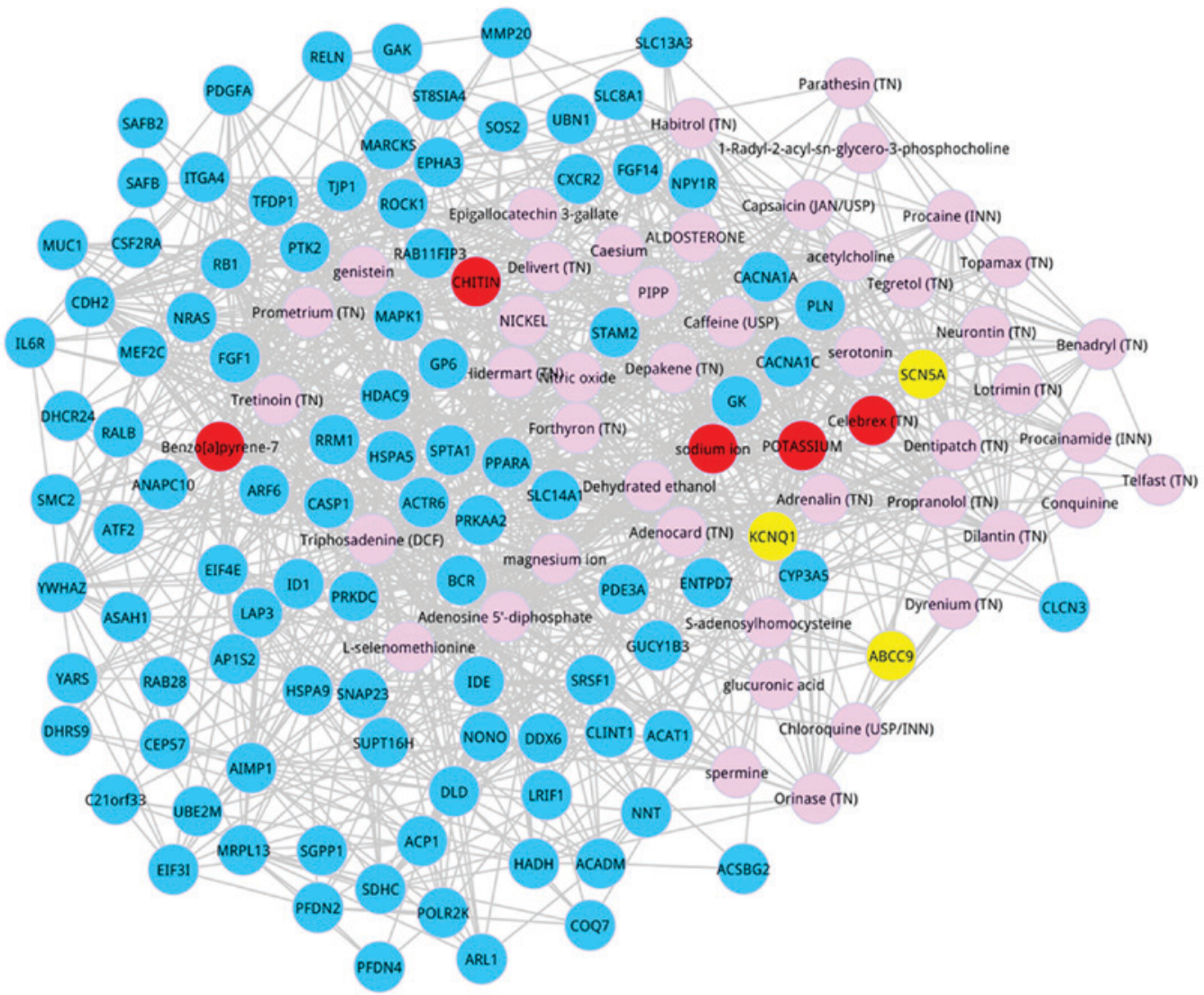

Figure 2. Construction of the co-expressed network. Blue nodes, top 100 co-expressed genes; yellow nodes, the seed genes; pink nodes, the metabolites; and red nodes, behalf of the top 5 metabolites. 
Prioritization of the risk metabolites of $A F$. There are 11 disease-related genes related to AF in OMIM, including ABCC9, GJA5, KCNA5, KCNE2, KCNJ2, KCNQ1, NPPA, $N U P 155, S C N 1 B, S C N 2 B$, and $S C N 5 A$, which were extracted and defined as seed genes. Nevertheless, no known disease metabolite data on AF were deposited in HMDB. In our work, the whole metabolome as candidates, the phenotype of AF and the 11 disease genes were utilized as seeds. With the goal of illustrating the intrinsic mode of this method, we sorted the metabolites of the composite network in descending order based on the interaction scores. The top 50 metabolites were dissected, as shown in Table III. The top 5 metabolites were respectively potassium (score $=0.004268$ ), sodium ion (score $=0.002251)$, Chitin (score $=0.001765)$, Benzo[a]pyrene-7,8dihydrodiol-9,10-oxide (score $=0.001509)$, and Celebrex $(\mathrm{TN})$ (score $=0.001131)$. A subnetwork was detected from the whole composite network in which the top 50 metabolites were included (Fig. 1). Based on the degree analysis for the entire composite network, we found that potassium had the highest degree (degree $=42$ ), and sodium ion also possessed the higher degree (degree $=39$ ).

Subsequently, we identified the co-expressed genes which interacted with the top 50 metabolites based on the score ranking. Based on the defined condition, we identified the top 100 genes, and the co-expressed network of the top 100 genes and the top 50 metabolites are shown in Fig. 2. After degree analysis for the co-expressed network, 4 metabolites possessed the degree $>50$, including adenosine 5 '-diphosphate (degree $=58)$, magnesium ion (degree $=57)$, potassium (degree $=52$ ), and sodium ion (degree $=52$ ).

\section{Discussion}

AF is the most common cardiac arrhythmia, but the molecular metabolites remain undefined. To the best of our knowledge, the present study was the first to compare the expression of metabolites between both atria in AF and SR. Based on the results, we found that the top metabolites of potassium and sodium ion had the highest degrees in the composite network and co-expressed network.

Potassium is reported to be connected with a higher risk of cardiovascular disease, for example, ventricular arrhythmias and cardiac arrest (19). Many studies have suggested that increased inward-rectifier $\mathrm{K}+$ current (IK1) is a prominent feature of atrial electrical remodeling (20-22). Moreover, AF is a final common endpoint of atrial remodeling (23). Olesen et al (24) demonstrated that enhanced potassium current increases AF susceptibility and Linz et al (25) demonstrated that blocking the activated atrial potassium currents inhibits AF in a pig model of obstructive apnea. Accordingly, we infer that the changes of potassium during AF might contribute to the self-perpetuating nature of the arrhythmia.

Another metabolite of sodium ion also had the higher degree in our study.

Sodium current is responsible for the early fast depolarization upstroke of the cardiac action potential (26). Reduced sodium current has been demonstrated to shorten the refractory period, and then to create a substrate for re-entry, thereby contributing to AF susceptibility $(27,28)$. Importantly, decreased sodium current may slow the electric conduction, and electrical remodeling plays important roles in the development of AF (29). Moreover, AF-related mutations have been found in sodium ion channel subunits, for example, $S C N 5 A$, $S C N 1 B$, and $S C N 2 B$ (30). Thus, we speculate that sodium ion is highly associated with the progression of AF.

Our work is the first to implement the analysis on AF by identifying the metabolites through systematically integrating multi-data including metabolic, phenomic, and genomic information. This is the main strong advantage of our work. We successfully detected several significant metabolites according to this computational method. Nevertheless, in the process of our study, we carried out an in-depth analysis using extremely well-matched but small size samples. The evaluation of the influence of metabolism underlying AF progression will need further exploration based on larger independent data sets. Moreover, our study merely fixed on analysis based on the bioinformatics, but the findings were not proven by the experiments. Further, this work is based on the gene expression in transcriptional level, not protein level. Consequently, further validation studies and functional experiments are required to confirm the significance of these initial discoveries. Despite these limitations, we believe that the prioritized metabolites can provide researchers valuable information for focusing research efforts to explore the molecular mechanisms of disease, and extracting potential bio-signatures for diagnosis and treatment of AF.

In a nutshell, metabolites such as potassium and sodium ion might be potential biomarkers for AF. Moreover, these metabolites might provide worthy clues for early diagnostic and therapeutic targets for AF.

\section{Acknowledgements}

Not applicable.

\section{Funding}

This study was supported by a grant from the National Natural Science Foundation of China: No. 81460055.

\section{Availability of data and materials}

The datasets used and/or analyzed during the current study are available from the corresponding author on reasonable request.

\section{Authors' contributions}

ZTY and JMH designed the study, revised the figures and drafted the manuscript. WLL, JWL and KZ acquired and analyzed the data, and prepared the figures. All authors read and approved the final manuscript.

\section{Ethics' approval and consent to participate}

Not applicable.

\section{Patient consent for publication}

Not applicable. 


\section{Competing interests}

The authors declare that they have no competing interests.

\section{References}

1. Mahida S: Transcription factors and atrial fibrillation. Cardiovasc Res 101: 194-202, 2014.

2. Santulli G and Totary-Jain H: Tailoring mTOR-based therapy: Molecular evidence and clinical challenges. Pharmacogenomics 14: 1517-1526, 2013.

3. Thomas IC and Sorrentino MJ: Bleeding risk prediction models in atrial fibrillation. Curr Cardiol Rep 16: 432, 2014.

4. Garg A and Akoum N: Atrial fibrillation and heart failure: Beyond the heart rate. Curr Opin Cardiol 28: 332-336, 2013.

5. Santulli G, Iaccarino G, De Luca N, Trimarco B and Condorelli G: Atrial fibrillation and microRNAs. Front Physiol 5: 15, 2014.

6. Fiehn O: Metabolomics - the link between genotypes and phenotypes. Plant Mol Biol 48: 155-171, 2002.

7. Nicholson JK and Wilson ID: Opinion: Understanding 'global' systems biology: Metabonomics and the continuum of metabolism. Nat Rev Drug Discov 2: 668-676, 2003.

8. Holmes E, Wilson ID and Nicholson JK: Metabolic phenotyping in health and disease. Cell 134: 714-717, 2008.

9. Ritchie MD, Holzinger ER, Li R, Pendergrass SA and Kim D: Methods of integrating data to uncover genotype-phenotype interactions. Nat Rev Genet 16: 85-97, 2015.

10. Blekherman G, Laubenbacher R, Cortes DF, Mendes P, Torti FM, Akman S, Torti SV and Shulaev V: Bioinformatics tools for cancer metabolomics. Metabolomics 7: 329-343, 2011.

11. Tsai FC, Lin YC, Chang SH, Chang GJ, Hsu YJ, Lin YM, Lee YS, Wang CL and Yeh YH: Differential left-to-right atria gene expression ratio in human sinus rhythm and atrial fibrillation: Implications for arrhythmogenesis and thrombogenesis. Int J Cardiol 222: 104-112, 2016.

12. Benjamini Y, Drai D, Elmer G, Kafkafi N and Golani I: Controlling the false discovery rate in behavior genetics research. Behav Brain Res 125: 279-284, 2001.

13. Xia J and Wishart DS: MSEA: A web-based tool to identify biologically meaningful patterns in quantitative metabolomic data. Nucleic Acids Res 38 (Web Server): W71-7, 2010.

14. Jewison T, Su Y, Disfany FM, Liang Y, Knox C, Maciejewski A, Poelzer J, Huynh J, Zhou Y, Arndt D, et al: SMPDB 2.0: Big improvements to the Small Molecule Pathway Database. Nucleic Acids Res 42: 478-484, 2014.

15. Kuhn M, Szklarczyk D, Franceschini A, Campillos M, von Mering C, Jensen LJ, Beyer A and Bork P: STITCH 2: An interaction network database for small molecules and proteins. Nucleic Acids Res 38: 552-556, 2010.

16. van Driel MA, Bruggeman J, Vriend G, Brunner HG and Leunissen JA: A text-mining analysis of the human phenome. Eur J Hum Genet 14: 535-542, 2006.

17. Yao Q, Xu Y, Yang H, Shang D, Zhang C, Zhang Y, Sun Z, Shi X, Feng L, Han J, et al: Global prioritization of disease candidate metabolites based on a multi-omics composite network. Sci Rep 5: 17201, 2015.
18. Wu X, Jiang R, Zhang MQ and Li S: Network-based global inference of human disease genes. Mol Syst Biol 4: 189, 2008.

19. Macdonald JE and Struthers AD: What is the optimal serum potassium level in cardiovascular patients? J Am Coll Cardiol 43: 155-161, 2004.

20. Atienza F, Almendral J, Moreno J, Vaidyanathan R, Talkachou A, Kalifa J, Arenal A, Villacastín JP, Torrecilla EG, Sánchez A, et al: Activation of inward rectifier potassium channels accelerates atrial fibrillation in humans: Evidence for a reentrant mechanism. Circulation 114: 2434-2442, 2006.

21. Gaborit N, Steenman M, Lamirault G, Le Meur N, Le Bouter S, Lande G, Léger J, Charpentier F, Christ T, Dobrev D, et al: Human atrial ion channel and transporter subunit gene-expression remodeling associated with valvular heart disease and atrial fibrillation. Circulation 112: 471-481, 2005.

22. Nattel S, Maguy A, Le Bouter S and Yeh YH: Arrhythmogenic ion-channel remodeling in the heart: Heart failure, myocardial infarction, and atrial fibrillation. Physiol Rev 87: 425-456, 2007.

23. Wakili R, Voigt N, Kääb S, Dobrev D and Nattel S: Recent advances in the molecular pathophysiology of atrial fibrillation. J Clin Invest 121: 2955-2968, 2011.

24. Olesen MS, Bentzen BH, Nielsen JB, Steffensen AB, David JP, Jabbari J, Jensen HK, Haunsø S, Svendsen JH and Schmitt N: Mutations in the potassium channel subunit KCNE1 are associated with early-onset familial atrial fibrillation. BMC Med Genet 13: 24, 2012.

25. Linz D, Schotten U, Neuberger H, Böhm M and Wirth K: Combined blockade of early and late activated atrial potassium currents suppresses atrial fibrillation in a pig model of obstructive apnea. Heart rhythm 8: 1933-1999, 2011.

26. Darbar D, Kannankeril PJ, Donahue BS, Kucera G, Stubblefield T, Haines JL, George AL Jr and Roden DM: Cardiac sodium channel (SCN5A) variants associated with atrial fibrillation. Circulation 117: 1927-1935, 2008.

27. Nattel S: New ideas about atrial fibrillation 50 years on. Nature 415: 219-226, 2002.

28. Terrenoire C, Simhaee D and Kass RS: Role of sodium channels in propagation in heart muscle: How subtle genetic alterations result in major arrhythmic disorders. J Cardiovasc Electrophysiol 18: 900-905, 2007

29. Opacic D, van Bragt KA, Nasrallah HM, Schotten U and Verheule S: Atrial metabolism and tissue perfusion as determinants of electrical and structural remodelling in atrial fibrillation. Cardiovasc Res 109: 527-541, 2016.

30. Watanabe H, Darbar D, Kaiser DW, Jiramongkolchai K, Chopra S, Donahue BS, Kannankeril PJ and Roden DM: Mutations in sodium channel $\beta 1$ - and $\beta 2$-subunits associated with atrial fibrillation. Circ Arrhythm Electrophysiol 2: 268-275, 2009.

(i) $\Theta$ This work is licensed under a Creative Commons Attribution-NonCommercial-NoDerivatives 4.0 International (CC BY-NC-ND 4.0) License. 